\title{
GEOMETRIC INFINITE DIVISIBILITY, STABILITY, AND SELF-SIMILARITY: AN OVERVIEW
}

\author{
TOMASZ J. KOZUBOWSKI \\ Department of Mathematics \& Statistics, University of Nevada \\ Reno, NV 89523, USA \\ E-mail: tkozubow@unr.edu
}

\begin{abstract}
The concepts of geometric infinite divisibility and stability extend the classical properties of infinite divisibility and stability to geometric convolutions. In this setting, a random variable $X$ is geometrically infinitely divisible if it can be expressed as a random sum of $N_{p}$ components for each $p \in(0,1)$, where $N_{p}$ is a geometric random variable with mean $1 / p$, independent of the components. If the components have the same distribution as that of a rescaled $X$, then $X$ is (strictly) geometric stable. This leads to broad classes of probability distributions closely connected with their classical counterparts. We review fundamental properties of these distributions and discuss further extensions connected with geometric sums, including multivariate and operator geometric stability, discrete analogs, and geometric self-similarity.
\end{abstract}

1. Introduction. The concepts of geometric stability and infinite divisibility, which goes back to [70], extends the classical properties of stability and infinite divisibility (ID) to geometric convolutions

$$
S_{p}=\sum_{j=1}^{N_{p}} X_{j},
$$

where $N_{p}$ is a geometric variable given by the probability density function (PDF)

$$
P\left(N_{p}=k\right)=p(1-p)^{k-1}, \quad k=1,2, \ldots
$$

while the $\left\{X_{j}\right\}$ are independent and identically distributed (IID) variables independent of $N_{p}$. In this context, the distribution of $X$ is geometrically infinitely divisible if for each

2010 Mathematics Subject Classification: Primary 60-02; Secondary 60E05, 60E07, 60F05, 60G18, 60G50, 60G51, 60G52, 62E10, 62E15, 62H05.

Key words and phrases: asymmetric Laplace distribution, fractional Brownian motion, gamma process, geometric distribution, geometric stable distribution, heavy tail, infinite divisibility, Lévy process, Linnik distribution, Mittag-Leffler distribution, operator stable distribution, random stability, random sum, scale mixture, self-similarity, stable distribution, subordination. The paper is in final form and no version of it will be published elsewhere. 
$p \in(0,1), X$ has the same distribution as $S_{p}$ (where of course the distribution of the $\left\{X_{j}\right\}$ depends on $p$ ). Similarly, the distribution of $X$ is (strictly) geometric stable if $X$ has the same distribution as $a_{p} S_{p}$ for some deterministic $a_{p}$, where this time the $\left\{X_{j}\right\}$ in (1) have the same distribution as $X$. This leads to broad classes of probability distributions, closely connected with their classical counterparts. A sharp peak at the mode, similar to that of the Laplace distribution (which is geometric stable), is one characteristic feature of these distributions, in addition to their heavy tails and infinite divisibility. These properties, coupled with their relation to geometric sums, which appear quite naturally in many applied problems (see, e.g., 68]), make these distributions applicable in many diverse areas.

This is a brief review of geometric stable and infinitely divisible distributions, as well as other interesting and important classes of distributions that arise in connection with geometric random sums (1). We emphasize general mechanisms generating these distributions, their fundamental properties that are relevant in applications, and their relation to other well-known classes, most notably, univariate and multivariate as well as operator stable laws. Our journey begins with Sections 2 and 3 , where we introduce the concepts of geometric stability and infinite divisibility. It continues with Section 4, where we review general geometric stable laws, which are the limiting distributions of geometric sums (1). Further extensions connected with multivariate geometric sums (1) normalized by linear operators are discussed in Section 5 . Our final destination is Section 6, devoted to quite recent concepts and stochastic models connected with geometric stability and self-similarity of random processes.

2. Geometric stability. The origins of (strictly) geometric stable distributions are connected with invariance properties of renewal process with respect to geometric thinning. Suppose that each point of a renewal process $\{R(t), t \geq 0\}$ is retained with probability $p \in(0,1)$ and deleted with probability $1-p$, independently of other points, leading to the new point process $\left\{R_{p}(t), t \geq 0\right\}$, known as the $p$-thinning of $R$ (see, e.g., [43]) or rarefaction (see [189]). Gnedenko [37] and Rényi [165, among others, characterized those renewal processes where $R$ and $R_{p}$ have the same distribution for each $p$ (up to the change of time scale). Since the inter-arrival of $R_{p}$ is the geometric sum (1) of $N_{p}$ inter-arrivals $X_{j}$ of $R$, we can express this invariance as the equality in distribution

$$
X \stackrel{d}{=} a_{p} \sum_{j=1}^{N_{p}} X_{j}, \quad p \in(0,1),
$$

where $a_{p}>0$ and $X, X_{1}, X_{2}, \ldots$ are IID, independent of $N_{p}$. Perhaps the best-known solution of (3), presented in [7, is the exponential distribution given by the Laplace transform (LT)

$$
\psi(t)=\frac{1}{1+\lambda t^{\alpha}}, \quad t>0,
$$

with $\alpha=1$ and $a_{p}=p$ in (3), and the corresponding renewal process $R$ is Poisson (see [165]). As shown in [37, the only other solution of (3) supported on the positive real line is that given by the LT (4) with some $0<\alpha \leq 1$ and $a_{p}=p^{1 / \alpha}$ in (3). This 
is the Mittag-Leffler distribution (see, e.g., [61, 62, 63, 80, 89, 90, 126, 156, 192]), also known as the positive Linnik law (see [128, 151]), which plays a crucial role in heavy-tail renewal theory arising in connection with anomalous (fractional) diffusion (see, e.g., 45] and references therein). The latter terminology has to do with the fact that distributions with the characteristic function $(\mathrm{ChF})$ given by

$$
\psi(t)=\frac{1}{1+\sigma^{\alpha}|t|^{\alpha}}, \quad t \in R,
$$

with $0<\alpha \leq 2$, known as (symmetric) Linnik distributions (see, e.g., 4, 5, 23, 67, 78, 79, 125. 127, 129, 150]), solve (3) within the class of symmetric distributions on the entire real line. Since the special case $\alpha=2$ yields the familiar Laplace distribution with its characteristic peak at the mode (see [77] and references therein), Linnik laws (5) are also known as $\alpha$-Laplace distributions (see [154]).

Note that the ChFs of all these distributions are related to those of stable distributions via the relation

$$
\psi(t)=\frac{1}{1-\log \phi(t)},
$$

where $\phi$ is the $\mathrm{ChF}$ of a degenerate distribution when $\psi$ is exponential, stable subordinator when $\psi$ is Mittag-Leffler, symmetric stable when $\psi$ is symmetric Linnik, and normal when $\psi$ is Laplace. These are all examples of strictly geometric stable (SGS) random variables, introduced in [70] as those variables that satisfy the stability relation (3). As shown in [70], the general class of SGS distributions is given by the ChF (6), where $\phi$ is the ChF of a strictly stable distribution (see, e.g., [172]). The same concept applies to distributions on $R^{d}$ (see, e.g., [118) or in general Banach spaces (see [160]). Let us also note that the relation (6) has an interpretation in terms of subordination, or randomly stopped Lévy processes: the ChF $\psi$ corresponds to the variable $X(Z)$, where $\{X(t), t \geq 0\}$ is a Lévy process with $X(1)$ having the $\mathrm{ChF} \phi$, and $Z$ is an independent exponential variable. It is clear in this connection that the distribution given by $\psi$ is infinitely divisible (ID), with the $\mathrm{ChF} \psi^{u}$ corresponding to the random variable $X(Z(u)$ ), with $X(t)$ as before and $\{Z(t), t \geq 0\}$ being the gamma Lévy process (so that $Z(1)$ is exponential). Special cases of random variables with $\mathrm{ChF} \psi^{u}$ include generalized Mittag-Leffler (see, e.g., [2]), generalized Linnik (see, e.g., [32, 152]), and generalized Laplace (or Bessel function) distributions (see, e.g., [77]).

REMARK 2.1. In case of exponential distribution, where in (3) we have $a_{p}=p$, relation (3) written in terms of the exponential $\mathrm{ChF} \psi$ takes on the form

$$
\psi(t)=\frac{p \psi(p t)}{1-(1-p) \psi(p t)},
$$

or equivalently, $\psi(t)=\psi(p t)(p+(1-p) \psi(t))$. This shows that an exponential variable $X$ solves the equation $X \stackrel{d}{=} W_{1} X_{1}+p X_{2}$, where $X, X_{1}$, and $X_{2}$ are IID and $W_{1}$ has a Bernoulli distribution with parameter $1-p=P\left(W_{1}=1\right)$, and is independent of $X_{1}$ and $X_{2}$. More general equations of the form $X \stackrel{d}{=} W_{1} X_{1}+W_{2} X_{2}$, with $\left(W_{1}, W_{2}\right)$ such that $E\left(W_{1}+W_{2}\right)=1$, were investigated in [9]. 
REMARK 2.2. The stability property (3) of the exponential distribution under geometric summation has been observed empirically in a variety of settings (see references in [108]), most notably in connection with studying growth periods of currency exchange rates (see [99, 109]). Within a growth period, all consecutive $\log$-returns $X_{j}=\log R_{j+1}-\log R_{j}$, where $R_{j}$ is the exchange rate on day $j$, are positive. Moreover, the cumulative log-return over the growth period is given by the random sum $S_{p}$ in 11 , where the number of terms $N_{p}$ is the duration of the growth period. A remarkable property of the exchange rates, reported in 99, 109, shows that cumulative log-return $S_{p}$ has the same distribution (up to a scale factor) as a single log-return $X_{j}$ (over one day) given that $X_{j}$ is positive. This is essentially the stability property (3). An interesting mixed bivariate model $\left(S_{p}, N_{p}\right)$, where the $N_{p}$ is geometric and the $\left\{X_{j}\right\}$ in (1) are IID exponential, was developed in this connection in 99] (see [11, 12, 100, 101, 102] for further extensions and applications of this model).

REMARK 2.3. The concept of geometric stability as defined in (3) needs a modification in order to be applicable to discrete distributions. Such modification appeared in [2, 14. where the multiplication by $a_{p}$ was replaced by the binomial thinning operator $\odot$, introduced in [188] in connection with extending the concepts of self-decomposability and stability to this setting. Recall that for any discrete random variable $N$ supported on the set of non-negative integers $Z_{+}=\{0,1, \ldots\}$ and any $a \in(0,1)$, we have

$$
a \odot N=\sum_{j=1}^{N} I_{j},
$$

where the $\left\{I_{j}\right\}$ are IID indicator random variables with parameter $a$, independent of $N$. In this setting, a $Z_{+}$-valued random variable $X$ is discrete strictly geometric stable (DSGS) if for each $p \in(0,1)$ there exist an $a_{p} \in(0,1)$ such that $X \stackrel{d}{=} a_{p} \odot S_{p}$, where $S_{p}$ is the sum (1) whose terms are IID copies of $X$, independent of the geometric number of terms $N_{p}$. As shown in [2], there is a correspondence between DSGS distributions and discrete stable laws (see [24, 188]), which can be stated as

$$
G(s)=\frac{1}{1-\log H(s)},
$$

where $G$ is the probability generating function (PGF) of a DSGS distribution and $H$ is the PGF of the corresponding discrete stable distribution. Since the PGF of any discrete stable distribution is of the form $H(s)=\phi(1-s)$, where $\phi$ is the LT of a stable subordinator (see [188]), the PGF of a DSGS distribution is of the form $\psi(1-s)$, where $\psi$ is the Mittag-Leffler LT given in (4). Note that this distribution, called discrete Mittag-Leffler in [60, 157, describes a standard Poisson process $\{N(t), t \geq 0\}$, randomly stopped at a Mittag-Leffler distributed $Z$, which is independent of the process. See [2, 3, 14, 15, 20, 57, 151, 179, 180] for further properties and extensions, including autoregressive schemes with discrete stationary distributions.

REMARK 2.4. The problem of random stability in the spirit of (3) was considered in many works, including [5, 16, 17, 41, 42, 54, 55, 67, 70, 72, 75, 125. It is now well-known that the geometric distribution is somewhat unique in that it allows the existence of distributions 
satisfying the stability relation (3). The key property of the geometric distribution that is behind this is the fact that the class $\left\{G_{p}, p \in(0,1)\right\}$ of geometric probability generating functions (PGF) forms a commutative semi-group under the operation of convolution: $G_{p}\left(G_{q}(s)\right)=G_{q}\left(G_{p}(s)\right)$ for all $p, q \in(0,1)$, where

$$
G_{p}(s)=\frac{p s}{1-(1-p) s}
$$

is the PGF of the geometric distribution (2). Indeed, a notion of stability with respect to a general family of integer-valued random variables $\left\{N_{p}, p \in \Delta \subset(0,1)\right\}$ requires that the corresponding PGFs commute (see [17, 42, 75]). Perhaps the easiest way to see this, is to note that if the distribution of $X$ is stable with respect to $N_{p}$ and $N_{q}$ for some $p, q \in \Delta$, so that for some $a_{p}, a_{q}>0$ we have

$$
X \stackrel{d}{=} a_{p} \sum_{j=1}^{N_{p}} X_{j} \text { and } X \stackrel{d}{=} a_{q} \sum_{j=1}^{N_{q}} X_{j},
$$

where the $\left\{X_{j}\right\}$ are IID copies of $X$, then we will also have

$$
X \stackrel{d}{=} a_{p} a_{q} \sum_{i=1}^{N_{p}} \sum_{j=1}^{N_{q}} X_{i j} \stackrel{d}{=} a_{q} a_{p} \sum_{i=1}^{N_{q}} \sum_{j=1}^{N_{p}} X_{i j},
$$

where the $\left\{X_{i j}\right\}$ are again IID copies of $X$, which essentially shows that $p q \in \Delta$ and $X$ is stable with respect to $N_{p q}$, whose PGF is $G_{p q}(s)=N_{p}\left(N_{q}(s)\right)=N_{q}\left(N_{p}(s)\right)$. There are very few such families with commuting PGFs, all related to the geometric distribution (see [17]). Thus, for most cases other than geometric, we do not have distributions with random stability in the spirit of (3). For example, no such stable distributions exist when $N_{p}$ is a Poisson distribution with mean $-\log p, p \in(0,1)$.

3. Geometric infinite divisibility. Geometric infinitely divisible random variables, introduced in [70, are those variables that can be decomposed into the sum of $N_{p}$ IID random variables for each $p \in(0,1)$, where $N_{p}$ is a geometric random variable.

Definition 3.1. A random variable in $R^{d}$ is geometrically infinitely divisible (GID) if for each $p \in(0,1)$ we have

$$
X \stackrel{d}{=} X_{1}^{(p)}+X_{2}^{(p)}+\cdots+X_{N_{p}}^{(p)}
$$

where $N_{p}$ is a geometric variable $\sqrt{2}$ and the $\left\{X_{j}^{(p)}\right\}$ are IID variables independent of $N_{p}$.

Writing equation 130 in terms of the ChFs we obtain

$$
\psi(t)=\frac{p \psi_{p}(t)}{1-(1-p) \psi_{p}(t)},
$$

where $\psi$ and $\psi_{p}$ are the ChFs of $X$ and $X_{1}^{(p)}$, respectively. This shows that in order for $X$ to be GID, the function $\psi_{p}(t)=\psi(t)[p+(1-p) \psi(t)]^{-1}$ must be a genuine ChF for each $p \in[0,1]$. Clearly, all strictly geometric stable distributions given by the ChF (6) are GID. As shown in [70, all GID distributions are given by the $\mathrm{ChF}$ of the form (6) with $\phi$ being the $\mathrm{ChF}$ of an ID distribution in the classical sense. Thus, there is a one-to-one correspondence between ID and GID distributions, as there is between SGS and strictly 
stable laws. Here, we again have an interpretation in terms of randomly stopped Lévy processes: the $\mathrm{ChF} \psi$ of a GID distribution corresponds to the variable $X(Z)$, where $\{X(t), t \geq 0\}$ is a Lévy process with $X(1)$ having the $\mathrm{ChF} \phi$, and $Z$ is an independent exponential variable. This shows that all GID distributions are also ID in the classical sense.

REMARK 3.2. Note that the relation (14) can be written as

$$
\psi(t)=\psi_{p}(t)\{p+(1-p) \psi(t)\},
$$

which in terms of random variables can be stated as

$$
X \stackrel{d}{=} I X+X^{(p)}
$$

where $X$ and $X^{(p)}$ are random variables with the ChFs $\psi$ and $\psi_{p}$, respectively, $I$ is a Bernoulli variable with $P(I=1)=1-p$, and all the variables on the right-handside of (16) are mutually independent. Thus, the solution of the problem of finding all random variables $X$ satisfying the relation (16), posed by Zolotarev (cf. [70]), is provided by the class of GID random variables. It is worth noting in this connection that only (and any) GID distribution can appear as the marginal distribution of $X_{n}$ in stationary autoregressive schemes of the form

$$
X_{n}= \begin{cases}\varepsilon_{n} & \text { with probability } p \\ \varepsilon_{n}+X_{n-1} & \text { with probability } 1-p,\end{cases}
$$

with $\varepsilon_{n}$ having the ChF $\psi_{p}$. Such models appeared in [5, 58, 59, 61, 122, 123, 184, 185, 186, 187, see also [112.

REMARK 3.3. When defining the concept of GID for discrete distributions supported on the set of non-negative integers $Z_{+}$, one may require that the distribution of the variables $\left\{X_{j}^{(p)}\right\}$ in 13 be also supported on $Z_{+}$(see [2]). In this case, the relation 6 is usually stated as in (9) in terms of the PGFs rather than ChFs (see [2]), where $G$ is the PGF of a discrete-GID distribution with $\mathrm{ChF} \psi$ and $H$ is the PGF of the corresponding discrete-ID distribution with $\mathrm{ChF} \phi$. Since all discrete-ID distributions are compound Poisson, so that $H(s)=\exp \{-\lambda[1-Q(s)]\}$ with some $\lambda>0$ and PGF $G$, every discrete-GID PGF admits the representation

$$
G(s)=\frac{1}{1+\lambda[1-Q(s)]} .
$$

Since the right-hand-side of 18 is the PGF of $\sum_{j=1}^{N_{p}-1} W_{j}$, where $N_{p}$ is geometric 2 with the parameter $p=1 /(1+\lambda)$ and the $\left\{W_{j}\right\}$ are IID with common PGF $Q$, and are independent of the $N_{p}$, we see that every discrete-GID distribution is compound geometric, as established in [2].

It is worth noting that the geometric random variable (2) is not GID (see 99]), while a shifted geometric random variable $N_{p}-1$ is GID. Further, if $X$ with the ChF $\psi$ is GID, then the variable $X^{(p)}$ with the $\mathrm{ChF} \psi_{p}$ appearing in 14 is also GID for each $p \in(0,1)$. In addition, the class of GID distributions is closed under geometric summation, that is the geometric sum $S=\sum_{j=1}^{N_{q}} X_{j}$ is GID whenever the $X_{j}$ are IID and GID and $N_{q}$ is geometric with parameter $q$, and independent of the $\left\{X_{j}\right\}$. This can be seen by noting 
that whenever the $\mathrm{ChF}$ of $X_{j}$ is of the form (6) with some ID $\phi$ then the ChF of $S$ is also of this form but with $\phi$ replaced with $\phi^{1 / q}$ (which is still ID). Let us also note that any mixture of a GID distribution with a point mass at zero is always GID, that is for each $q \in(0,1)$ the $\mathrm{ChF} q+(1-q) \psi(t)$ is GID whenever $\psi$ is GID, although the converse does not hold (the mixture can still be GID with $\psi$ not being GID), see 114 for examples and further information. Finally, it should be noted that the class of GID distributions arises as the only limiting laws of geometric sums for triangular arrays, in a similar way as classical ID distributions arise in connection with deterministic sums. We refer the reader to [69, 146, 155, 158, 114, 173, 174, 175] for more information on GID, and to [2, 17, 42, 56, 75, 177, 178, 181] for generalizations to divisibility with respect to integer-valued distributions other than geometric.

4. Geometric stable distributions. With analogy to classical stable distributions, one can define a class of limiting distributions in the geometric summation scheme as follows (see [81, 142]).

Definition 4.1. A random vector $\mathrm{Y}$ in $R^{d}$ is said to be geometric stable (GS) with respect to the summation scheme if there exists a sequence of IID random vectors $X_{1}, X_{2}, \ldots$, a geometric random variable $N_{p}$ independent of all $X_{i}$, and constants $a_{p}>0$ and $b_{p} \in R^{d}$ such that

$$
a_{p} \sum_{i=1}^{N_{p}}\left(X_{i}+b_{p}\right) \stackrel{d}{\longrightarrow} Y, \quad \text { as } \quad p \rightarrow 0 .
$$

As shown in [71, GS distributions can be described in terms of their ChF via (6), where $\phi$ is a stable $\mathrm{ChF}$. If the stable $\mathrm{ChF} \phi$ corresponds to a strictly stable random vector, then $Y$ is called strictly geometric stable (SGS), and appears as a weak limit in 19. with $b_{p}=0$. If the distribution of a GS random variable $Y$ is symmetric, then $Y$ is called symmetric GS, and is necessarily SGS.

REMARK 4.2. One dimensional strictly GS laws defined as limiting distributions in 19 with $b_{p}=0$ coincide with those mentioned in Section 2 in connection with the stability of geometric sums.

REMARK 4.3. The characteristic function representation of GS distributions follows from classical transfer theorems for random sums that go back to Robbins [167, 168], see, e.g., [27, 38, 39, 40, 42, 95, 96, 120, 169, 171, 190. These results imply that whenever the $\left\{X_{i}\right\}$ are in the domain of attraction of a stable distribution with the ChF $\phi$ and the integer-valued random variables $N_{p}$ converge to infinity in probability (as $p$ converges to zero) such that $p N_{p}$ converges in distribution to a non-negative random variable $Z$ with cumulative distribution function $(\mathrm{CDF}) F$ and $\operatorname{LT} \varphi$, and we have the convergence in (19), then the $\mathrm{ChF}$ of the limit is the convolution mixture

$$
\psi(t)=\int_{0}^{\infty} \phi^{z}(t) d F(z)=\varphi(-\log \phi(t)) .
$$

In the geometric case, $p N_{p}$ converges to the standard exponential distribution with the LT $\varphi(t)=1 /(1+t)$, which produces the basic relation (6). Different families of distributions 
arise for other integer-valued variables $\left\{N_{p}, p \in \Delta \in(0,1)\right\}$, whose generating functions do not need to be commutative, which is required to ensure the stability as discussed in Section 2. More information related to this can be found in [42, 75, 95, 96].

REMARK 4.4. If the convergence in $(19)$ is along a particular subsequence, the resulting limiting distributions are called semi-geometric stable, with analogy to the semi-stable case. Their ChFs are still of the form (6), where this time $\phi$ is the ChF of a semi-stable law (see, e.g., [119, 153]). See [13, 26, 51, 60, 61, 63, 134, 135, 146, 154, 175] for further details.

4.1. GS characteristic function. A substitution of the stable $\mathrm{ChF}$ as given in 172 into (6) produces a GS ChF (see, e.g., [118]),

$$
\psi(t)=\left[1+\int_{S_{d}}|\langle t, s\rangle|^{\alpha} \omega_{\alpha, 1}(\langle t, s\rangle) \gamma(d s)-i\langle t, m\rangle\right]^{-1},
$$

where $\langle\cdot, \cdot\rangle$ denotes the inner product, $0<\alpha \leq 2, \gamma$ is a finite measure on the unit sphere $S_{d}$ of $R^{d}, m \in R^{d}$, and

$$
\omega_{\alpha, \beta}(x)= \begin{cases}1-i \beta \operatorname{sign}(x) \tan (\pi \alpha / 2), & \text { if } \alpha \neq 1, \\ 1+i \beta \frac{2}{\pi} \operatorname{sign}(x) \log |x|, & \text { if } \alpha=1 .\end{cases}
$$

As in the stable case, the parameter $\alpha$ is the index of stability that controls the tails, while $\gamma$ is the spectral measure that reflects the dependence structure. For example, the association of the the components of a GS random vector implies certain conditions on the spectral measure (see [124]). However, in contrast with the stable case, the components can never be independent and the location parameter $m$ is not a shift parameter, although it coincides with the mean whenever it exists $(\alpha>1)$. If $d=1$, the ChF can be written as (see, e.g., [117])

$$
\psi(t)=\left(1+\sigma^{\alpha}|t|^{\alpha} \omega_{\alpha, \beta}(t)-i \mu t\right)^{-1},
$$

where $\beta \in[-1,1]$ is a skewness parameter, while $\mu \in R$ and $\sigma \geq 0$ control location and scale, respectively. There are many other parameterizations of GS distributions, which parallel those for the stable case (see, e.g., [117]). Let us note that univariate GS distributions are supported on the entire real line, with the exception of the case $\alpha<1$ and $|\beta|=1$, where we obtain a distribution concentrated on $(0, \infty)$ for $\beta=1, \mu>0$ and on $(-\infty, 0)$ for $\beta=-1, \mu<0$. More information on the univariate case can be found in 10, 36, 73, 69, 83, 84, 87, 88, 117.

4.2. Basic properties. The following fundamental representation, taken from [97], shows that GS distributions are location and scale mixtures of stable distributions.

Proposition 4.5. If $Y$ is GS with index $\alpha$, spectral measure $\gamma$ and a location parameter $m$, and $X$ is $\alpha$-stable with the same spectral measure and location parameter 0 , then

$$
Y \stackrel{d}{=} \begin{cases}m Z+Z^{1 / \alpha} X, & \text { if } \alpha \neq 1, \\ m Z+\left(\frac{2}{\pi} Z \log Z\right) g+Z X, & \text { if } \alpha=1,\end{cases}
$$

where $Z$ is a standard exponential random variable, independent of $X$, and

$$
g=\left(g_{1}, \ldots, g_{d}\right)=\int_{S_{d}} s \gamma(d s)
$$


Many properties of GS distributions, such as their densities and distribution functions, moments, and tail behavior, follow from the stable case via 24. In particular, for any nonnegative $p_{1}, \ldots p_{d}$, the joint moment $E\left\{\left|Y_{1}\right|^{p_{1}} \cdots\left|Y_{d}\right|^{p_{d}}\right\}$ is finite if and only if $p_{1}+\cdots+$ $p_{d}<\alpha$, whenever $\alpha \in(0,2)$ and the coordinates of $Y=\left(Y_{1}, \ldots, Y_{d}\right)$ are $d$-fold dependent (see, e.g., [172]), that is $\gamma\left\{s \in S_{d}: s_{i} \neq 0, i=1, \ldots d\right\}>0$. In one dimension, we simply have $E\left[|Y|^{p}\right]<\infty$ if and only if $p<\alpha$. Moreover, for any Borel set $B \subset S_{d}$, we have $x^{\alpha} P(\|Y\|>x, Y /\|Y\| \in B) \rightarrow c_{\alpha} \gamma(B)$ as $x \rightarrow \infty$, where $c_{\alpha}$ is an appropriate constant. The individual coordinates of $Y$ have power tails as well, $x^{\alpha} P\left( \pm Y_{k}>x\right) \rightarrow c_{\alpha}\left(1 \pm \beta_{k}\right) \sigma_{k}^{\alpha} / 2$ as $x \rightarrow \infty$, where $\beta_{k}$ and $\sigma_{k}$ are the skewness and the scale parameters of $Y_{k}, k=1, \ldots d$. The PDFs and distribution functions of general GS distributions do not have explicit forms, but admit certain integral representations (obtained via (24), involving their stable counterparts. It should be noted that all marginals and linear transformations of GS random vectors are GS. In particular, the quantity $\langle b, Y\rangle$ is GS for all $b \in R^{d}$ whenever $Y$ is GS. The converse is also true when for all $b \in R^{d}$ the quantity $\langle b, Y\rangle$ is strictly GS, symmetric GS, or GS with $\alpha \in[1,2)$; the case $\alpha=2$ is still open.

Series representations in one dimension, analogous to LePage representations of stable random variables (see [172]), were developed in [104]. Information on simulation and parameter estimation connected with this model can be found in [82, 88, 98. There are also alternative representations (see [10, 25, 78, 86, 87, 152]), in particular, one showing that in one-dimension we always have $Y \stackrel{d}{=} Z T$, where $Z$ and $T$ are independent, $Y$ is GS, $Z$ is standard exponential, and $T$ has an explicit density (see 69]). Thus, all univariate GS distributions are exponential mixtures, and thus unimodal with the mode at the origin. Note that this property implies that all multivariate GS distributions are (linearly) unimodal with the mode at zero. Let us note that the unimodality of this class does not follow from their self-decomposability, as is the case with the stable laws, since not all GS distributions are in the class L of self-decomposable distributions (see, e.g., [163] for examples). It is also important to note that the multivariate GS densities in the symmetric case are unbounded at the mode, as are one-dimensional strictly GS densities with $\alpha<1$ (see [69]). This behavior at the mode is one characteristic feature of this class that distinguishes these distributions from the stable laws, which have the same tail behavior and domains of attraction. Since high peak at the mode and power tails are often found in empirical distributions of financial data, GS-based models offer an attractive alternative to normal and stable distributions in this area (see, e.g., [19, 81, 89, 92, 97, 103, 107, 109, 116, 130, 131, 143, 144, 159, 161, 162,).

4.3. Special cases and examples. Below we discuss important special cases of GS laws.

4.3.1. Improper GS laws. If the $\mathrm{ChF} \phi$ that appears in $(6)$ corresponds to a degenerate stable law, we obtain an improper GS distribution with the ChF

$$
\psi(t)=[1-i\langle t, m\rangle]^{-1},
$$

In one dimension, this distribution is the familiar exponential, while in $R^{d}$ we have $Y \stackrel{d}{=} m Z \stackrel{d}{=} D(m) W$, where $Y$ is a GS variable with the ChF 26, $Z$ is standard exponential, $D(m)$ is a diagonal matrix with the elements of $m$ on its main diagonal, and 
$W=\left(W_{1}, \ldots, W_{d}\right)$ has a Marshall-Olkin exponential distribution, given by the survival function

$$
P\left(W_{1}>x_{1}, \ldots, W_{d}>x_{d}\right)=e^{-\max \left(x_{1}, \ldots, x_{d}\right)} .
$$

Since $Y$ satisfies the stability relation (3) with $a_{p}=p$, the improper GS distribution is strictly geometric stable.

4.3.2. Strictly GS laws. Strictly GS (SGS) distributions arise as the limiting laws in (19) with $b_{p}=0$. Equivalently, SGS random vectors are characterized via the stability property (3), where necessarily $a_{p}=p^{1 / \alpha}$ (see, e.g., [84, 85]). As shown in [142], the ChF of a SGS random vector is of the form (6) with $\phi$ being the ChF of a strictly stable distribution. Consequently, all SGS distributions have the ChFs of the form (21), where either $\gamma \equiv 0$ (an improper GS law with the $\mathrm{ChF}(26)$ ) or $\gamma \neq 0$, in which case we must have $\alpha \neq 1$ and $m=0$ or $\alpha=1$ and $\int_{S_{d}} s_{k} \gamma(d s)=0$ for all $k=1, \ldots, d$. In one dimension, SGS distributions are described by the ChF (23) with $\alpha \neq 1$ and $\mu=0$ or $\alpha=1$ and $\beta=0$, although certain alternative parameterizations are often used in this case (see, e.g., [117]). These distributions are also known in the literature as the (non-symmetric) Linnik distributions (see, e.g., [29, 30, 31, 78]).

REMARK 4.6. Since all SGS random vectors $X$ are GID and the relation (14) holds with $\psi_{p}(t)=\psi\left(p^{1 / \alpha} t\right)$, where $\psi$ is the $\mathrm{ChF}$ of $X$, we also have 15 , which in this case can be written as

$$
\psi(t)=\psi(\rho t) \psi^{(\rho)}(t)
$$

with $\psi^{(\rho)}(t)=\rho^{\alpha}+\left(1-\rho^{\alpha}\right) \psi(t)$ and $\rho=p^{1 / \alpha} \in(0,1)$. What this shows is that all SGS distributions belong to the class L of the self-decomposable (SD) distributions, and lead to stationary, non-Gaussian autoregressive processes $X_{n}=\rho X_{n-1}+\varepsilon_{n}$, where the $\left\{X_{n}\right\}$ are SGS with common $\mathrm{ChF} \psi$ and the IID $\left\{\varepsilon_{n}\right\}$ have common $\mathrm{ChF} \psi^{(\rho)}$ (see [112]). Many such processes with general SD stationary distributions have been developed, including GS special cases of exponential processes (see, e.g., 35]), Laplace processes (see [1, 22, 121]), Linnik processes (see [5, 58]), and Mittag-Leffler processes (see [61]).

4.3.3. Symmetric GS laws. A GS random vector is called symmetric geometric stable if $P(Y \in A)=P(-Y \in A)$ for all Borel sets $A$ in $R^{d}$. It is well-known (see, e.g., 69]) that the $\mathrm{ChF}$ of symmetric GS random vectors with index $0<\alpha<2$ is of the form

$$
\psi(t)=\left[1+\int_{S_{d}}|\langle t, s\rangle|^{\alpha} \gamma(d s)\right]^{-1},
$$

where $\gamma$ is a unique, finite, and symmetric measure on $S_{d}$. When $\alpha=2$, the relation (27) holds as well, although in this case $\gamma$ is not unique. In one dimension, these are symmetric Linnik distributions with the $\mathrm{ChF}$ (5).

4.3.4. Multivariate Linnik distributions. If the $\mathrm{ChF} \phi$ in (6) corresponds to a subGaussian stable law on $R^{d}$ (see. e.g., [172]), then the corresponding GS ChF is of the form

$$
\psi(t)=\left[1+\left(\frac{1}{2} t^{\prime} \Sigma t\right)^{\alpha / 2}-i\langle m, t\rangle\right]^{-1},
$$


where $t^{\prime}$ denotes the transpose of $t$ and $\Sigma$ is a non-negative definite symmetric matrix. In the symmetric case $m=0$ we obtain the multivariate Linnik distribution studied in [4, 150, which generalizes the one-dimensional case (5).

4.3.5. Laplace distributions. If $\alpha=2$ then the GS ChF (21), as well as as the multivariate Linnik ChF 28, become

$$
\psi(t)=\left[1+\frac{1}{2} t^{\prime} \Sigma t-i\langle t, m\rangle\right]^{-1}
$$

where $\Sigma$ is a $d \times d$ non-negative definite symmetric matrix. If the matrix $\Sigma$ is positivedefinite, then the distribution is truly $d$-dimensional, and its PDF is expressible in terms of the modified Bessel functions of the third kind (see [106]). This multivariate asymmetric Laplace (AL) distribution (see [77, 106]), along with the improper one, stand out within the class of GS distributions in that they have a finite covariance matrix (with the AL covariance matrix being $\Sigma+m m^{\prime}$ ). In case $d=2 r+3$, where $r$ is a non-negative integer, the AL density takes on an explicit form (see [77]), which is particularly simple when $d=3(r=0)$ :

$$
g(y)=\frac{e^{-y^{\prime} \Sigma^{-1} m-\sqrt{2+m^{\prime} \Sigma^{-1} m} \sqrt{y^{\prime} \Sigma^{-1} y}}}{2 \pi \sqrt{y^{\prime} \Sigma^{-1} y}|\Sigma|^{1 / 2}}, y \neq 0 .
$$

It is worth noting that when $d>1$ all AL PDFs blow out at the origin, as is the case with multivariate symmetric GS densities as well. All marginal distributions of multivariate AL laws are also AL, as are their linear transformations. In addition, according to Proposition 4.5. all AL laws are location and scale mixtures of Gaussian distributions, as the variable $X$ in 24 in this case is Gaussian (with mean 0 and covariance $\Sigma$ ). In the symmetric case $m=0$, mentioned in [4, AL distributions are elliptically contoured, and belong to the class of $G$-type distributions. The latter are scale mixtures of normal laws with infinitely divisible stochastic variance (see [132, 133, 136, 170]).

When $d=1$, we obtain a univariate skew Laplace distribution (see [77, 103, 105, 107, 111, 112]) with the $\mathrm{ChF}$

$$
\psi(t)=\left(\frac{1}{1+i \frac{\sigma \kappa}{\sqrt{2}} t}\right)\left(\frac{1}{1-i \frac{\sigma}{\sqrt{2} \kappa} t}\right), t \in R, \kappa, \sigma>0,
$$

which reduces to the classical symmetric Laplace distribution with mean zero and variance $\sigma^{2}$ when the skewness parameter $\kappa$ is equal to one. The above factorization of the AL $\mathrm{ChF} \psi$ shows that an $\mathrm{AL}$ random variable $Y$ admits stochastic representations

$$
Y \stackrel{d}{=} \frac{1}{\sqrt{2}}\left(\frac{1}{\kappa} Z_{1}-\kappa Z_{2}\right) \stackrel{d}{=} \frac{1}{\sqrt{2}} I Z,
$$

where $Z, Z_{1}$ and $Z_{2}$ are IID standard exponential variables and $I$, which is independent of $Z$, takes on the values $1 / \kappa$ and $-\kappa$ with probabilities $1 /\left(1+\kappa^{2}\right)$ and $\kappa^{2} /\left(1+\kappa^{2}\right)$, respectively. Consequently, the $\mathrm{PDF}$ of $Y$ is of the form

$$
g(x)=\frac{2 \kappa}{1+\kappa^{2}} \cdot \begin{cases}f(x \kappa) & \text { for } x \geq 0 \\ f\left(\frac{x}{\kappa}\right) & \text { for } x<0\end{cases}
$$

where $f(x)=\exp \{-\sqrt{2}|x| / \sigma\} /(\sqrt{2} \sigma)$ is the PDF of the symmetric Laplace distribution 
with variance $\sigma^{2}$. Thus, the AL model emerges from a general scheme 32 of incorporating skewness into any symmetric PDF $f$, proposed in [34] (see also 33]). Many standard distributions were extended to their skew counterparts through this scheme, including normal (see [149]), student-t (see 6, 34, 44]), Weibull (see 65, 66]), and exponential power (see [8, 33, 76]).

With their characteristic peak at the mode and semi-heavy tails, skewness, and relation to limit results for geometric sums, multivariate and univariate Laplace distributions have appeared in many applications due to their simplicity and flexibility, see [77] for more information and additional references.

REMARK 4.7. A related class of distributions, termed log-Laplace (LL) in [108, 109] and double-Pareto in 164, arises via $X=\exp (Y)$ with $Y$ being AL. These distributions appeared in connection with modeling under-reported data in [47, 52. Their densities are double-power laws of the form

$$
f(x)=\frac{1}{\delta} \frac{\alpha \beta}{\alpha+\beta} \cdot \begin{cases}(x / \delta)^{\beta-1} & \text { for } 0<x<\delta \\ (\delta / x)^{\alpha+1} & \text { for } x \geq \delta,\end{cases}
$$

where $\delta>0$ (scale) and $\alpha, \beta>0$ (tail parameters). Consequently, a log-log plot of $f$ produces a distinct "tent" shape, observed empirically in a variety of fields in connection with modeling growth rates in numerous applications, such as gross domestic product, stock prices, company sizes, physics and others (extensive references are available in [108, 109]). It is argued in [108, 109] that the emergence of the LL distributions in these settings is connected with their fundamental properties of stability with respect to geometric multiplication (see [74]), which reflect analogous properties of the Laplace distribution in the summation setting.

5. Operator geometric stable distributions. Operator geometric stable laws generalize GS distributions, as they arise as the limiting distributions in 19 when the sums are normed by linear operators on $R^{d}$ rather then by scalars $a_{p}>0$ (see [75, 92]).

Definition 5.1. A random vector $\mathrm{Y}$ on $R^{d}$ is operator geometric stable (OGS) if for $N_{p}$ geometric with the PDF (2) there exist IID random vectors $X_{1}, X_{2}, \ldots$ independent of $N_{p}$, linear operators $A_{p}$, and centering constants $b_{p}$ such that

$$
A_{p} \sum_{i=1}^{N_{p}}\left(X_{i}+b_{p}\right) \stackrel{d}{\rightarrow} Y \quad \text { as } p \rightarrow 0 .
$$

If (34) holds we say that the distribution of $X_{1}$ is weakly geometrically attracted to that of $Y$, and the collection of such distributions is called the generalized domain of geometric attraction of $Y$.

As shown in 92, OGS distributions are all ID as well as GID, and are closely related to operator stable (OS) laws, which are the limiting distributions in this setting for deterministic sums (see, e.g., 64, 139]). Similarly to the GS case, any OGS ChF is of the form (6) with $\phi$ being operator stable ChF. Equivalently, any OGS random vector $Y$ has the same distribution as that of $L(Z)$, where $Z$ is a standard exponential variable while $\{L(t), t \geq 0\}$ is a stochastic process with stationary independent increments, independent 
of $Z$, and such that $L(1)$ is OS and $L(0)=0$ almost surely. Thus, every OGS random vector admits the stochastic representation

$$
Y \stackrel{d}{=} Z^{E} X+a_{Z}
$$

where $Z$ is as before and $X$, which is independent of $Z$, is OS with exponent $E$ and distribution $\omega$ satisfying

$$
\omega^{t}=t^{E} \omega * \varepsilon_{a_{t}} \quad \text { for all } t>0 .
$$

Here, $\varepsilon_{a}$ is the unit mass at the point $a \in R^{d}, t^{E}=\exp (E \log t)$ and $\exp (A)=I+$ $A+A^{2} / 2 !+A^{3} / 3 !+\cdots$ is the usual exponential operator. The representation (35) is a generalization of (24), which applies to GS random vectors.

REMARK 5.2. Using the results of [182] for subordinated Lévy processes (see also [53]), one can derive the Lévy representation of the OGS ChF. As shown in 92 the ChF of an OGS law, related to a full OS law $\omega$ via (6), is of the form

$$
\psi(t)=e^{i\langle a, t\rangle}+\int_{R^{d}}\left\{e^{i\langle t, x\rangle}-1-\frac{i\langle t, x\rangle}{1+\|x\|^{2}}\right\} d \nu(x)
$$

where $\nu(x)=h(x) d x, h(x)=\int_{0}^{\infty} g(s, x) \frac{1}{s} d e^{-s} d s$,

$$
a=\int_{0}^{\infty} \int_{R^{d}} \frac{x}{1+\|x\|^{2}} g(s, x) d x \frac{1}{s} e^{-s} d s,
$$

and $g(s, x)$ is the Lebesgue density of $\omega^{s}$ for any $s>0$. The asymptotics of the Lévy measure in the one-dimensional GS case were studied in [115].

REMARK 5.3. The problems of geometric summation (and geometric stability) with operator norming have also appeared in more abstract setting of probability on groups, see [48, 49, 50, 51] for details. Further generalizations where the random summation in (34) is other than geometric can be found in 93 .

5.1. Special cases and examples. Clearly, when the operators in $(34)$ are of the form $A_{p}=a_{p} I_{d}$, where $a_{p}>0$ and $I_{d}$ is a $d$-dimensional identity matrix, then the limiting distributions coincide with the GS laws on $R^{d}$. Additionally, we get multivariate Laplace distributions if the $\left\{X_{i}\right\}$ in 34 are in the normal domain of attraction.

5.1.1. Strictly OGS laws. If the OS law given by the characteristic function $\phi$ in (6) is strictly OS, then the distribution given by $\psi$ is called strictly OGS. For the strictly OGS distributions, the convergence in (34) holds with $b_{p}=0$. In addition, the representation (35) reduces to

$$
Y \stackrel{d}{=} Z^{E} X
$$

where, as before, $Z$ is a standard exponential variable and $X$ is strictly OS with exponent $E$ (and independent of $Z$ ). It should be noted that strictly OGS distributions generalize the stability property of the GS laws, see 92] (and also [49, 50] for more general results on strict geometric stability on nilpotent Lie groups). 
Proposition 5.4. Let $Y, Y_{1}, Y_{2}, \ldots$ be IID random variables in $R^{d}$, and let $N_{p}$ be a geometrically distributed random variable (2), independent of the sequence $\left(Y_{i}\right)$. Then

$$
S_{p}=A_{p} \sum_{i=1}^{N_{p}} Y_{i} \stackrel{d}{=} Y, \quad p \in(0,1),
$$

with some operators $A_{p}$ on $R^{d}$ if and only if $Y$ is strictly $O G S$, in which case $Y$ admits the representation (38) for some $O S$ random variable $X$ with exponent $E$ and $A_{p}=p^{E}$ for $p \in(0,1)$.

5.1.2. Marginally geometric stable laws. If the operators $A_{p}$ in (34) are diagonal matrices $\operatorname{diag}\left(a_{p, 1}, \ldots, a_{p, d}\right)$ for some positive $\left\{a_{p, i}\right\}$, then the one-dimensional marginals of the limiting OGS vector $Y$ are GS with the ChF (23) and possibly different values of $\alpha$. Here, the $\mathrm{ChF}$ of $Y$ is given by (6) with $\phi$ corresponding to a marginally stable OS random vector (see, e.g., [18, 137, 145, 166]). If the values of $\alpha$ for all marginal distributions are less than 2, then the ChF of $Y$ is of the form

$$
\psi(t)=\left(1+\int_{S_{d}} \int_{0}^{\infty}\left(e^{i\left\langle t, r^{E} s\right\rangle}-1-\frac{\left.i\left\langle t, r^{E} s\right)\right\rangle}{1+\left\|r^{E} s\right\|^{2}}\right) \frac{d r}{r^{2}} \gamma(d s)-i\langle t, m\rangle\right)^{-1},
$$

where $E$ is a diagonal matrix

$$
E=\operatorname{diag}\left(1 / \alpha_{1}, \ldots, 1 / \alpha_{d}\right), \quad 0<\alpha_{i} \leq 2, \quad i=1, \ldots, d,
$$

called the exponent of $Y, r^{E}=\operatorname{diag}\left(\left|r_{1}\right|^{1 / \alpha_{1}} \ldots,\left|r_{d}\right|^{1 / \alpha_{d}}\right)$, the spectral measure $\gamma$ is a finite measure on the unit sphere $S_{d}$ in $R^{d}$, and $m \in R^{d}$ is the shift parameter. As in the stable and GS cases, the measure $\gamma$ determines the dependence structure. The fact that these distributions allow for a different tail behavior for their marginals makes them, along with marginally stable laws, attractive in financial portfolio analysis (see 92, 140, 144, 159]). Let us note that OGS distributions admit a stability property similar to 39 with diagonal $A_{p}$ (see 92]). Two interesting examples from this class are a bivariate distribution with Laplace and Linnik marginal distribution (see [92]), given by the $\mathrm{ChF}$

$$
\psi(t, s)=\frac{1}{1+\sigma^{2} t^{2}+\eta^{\alpha}|s|^{\alpha}}, \quad s, t \in R, \alpha \in(0,2],
$$

and a bivariate distribution with exponential and Mittag-Leffler marginal distributions (see [91]), given by the LT

$$
\psi(t, s)=\frac{1}{1+\sigma t+\eta^{\alpha} s^{\alpha}}, \quad s, t \in R_{+}, \quad \alpha \in(0,1] .
$$

6. Geometric self-similarity. Recall that a stochastic process $X(t)$ is self-similar with index $H$ if for all $c>0$ we have $X(c \cdot) \stackrel{\mathrm{d}}{=} c^{H} X(\cdot)$, so that the time scale change is (stochastically) equivalent to the space scale change (see, e.g., 28]). In the same spirit, one can consider self-similarity with respect to a random time change, understood as subordination (see [94, 110]). Let $\mathcal{T}=\left\{T_{c}(t), t \geq 0\right\}, c \geq 1$, be a family of random time changes, with $T_{c}(0)=0$ almost surely and non-decreasing sample paths, such $E\left[T_{c}(t)\right]=$ ct. Following 94, we define stochastic self-similarity as follows. 
Definition 6.1. Let $\{X(t), t \geq 0\}$ be a stochastic process on $R^{d}$ and let $\mathcal{T}$ be a family of processes described above, independent of $X$. The process $X(t)$ is stochastically selfsimilar with index $H$ (or $H$-sss) with respect to the family $\mathcal{T}$ if

$$
X\left(T_{c}(\cdot)\right) \stackrel{d}{=} c^{H} X(\cdot), \quad c \geq 1 .
$$

REMARK 6.2. The above notion of stochastic self-similarity, which involves stochastic renormalization in time, is apparently unrelated to that considered in [46] and [191, which involves stochastic renormalization in space.

REMARK 6.3. Note that by iterating the relation (44), we obtain

$$
X\left(T_{c_{1}} \circ T_{c_{2}}(\cdot)\right) \stackrel{d}{=} X\left(T_{c_{2}} \circ T_{c_{1}}(\cdot)\right) \stackrel{d}{=}\left(c_{1} c_{2}\right)^{H} X(\cdot), \quad c_{1}, c_{2} \geq 1,
$$

so it is natural to require that the family $\mathcal{T}$ be a commutative composition semigroup, that is

$$
T_{c_{1}} \circ T_{c_{2}}(\cdot) \stackrel{d}{=} T_{c_{1} c_{2}}(\cdot), \quad c_{1}, c_{2} \geq 1,
$$

where $\circ$ stands for the composition of two functions.

6.1. Examples. One example of a process that is H-sss is the standard gamma process $\{G(t), t \geq 0\}$, which is stochastically self-similar (with index $H=1$ ) with respect to the family

$$
\mathcal{T}=\left\{T_{c}(t)=t+N B_{p}(t), t \geq 0\right\}, c \geq 1 .
$$

Here, $p=1 / c \in(0,1]$ and $\left\{N B_{p}(t), t \geq 0\right\}$ is a negative binomial Lévy process (NBP) studied in [113], whose marginal distributions are negative binomial with the PDF

$$
P\left(N B_{p}(t)=k\right)=\frac{\Gamma(t+k)}{\Gamma(t) k !} p^{t}(1-p)^{k}, \quad k=0,1,2, \ldots
$$

It is easy to verify that this family of negative binomial processes with drift satisfies all the required properties, including $E T_{c}(t)=c t$ as well as 46 .

REMARK 6.4. Another family of random time changes that satisfies these properties can be constructed as follows (see [110]). For each non-negative integer $k$ let

$$
N_{p}^{(k)}=k N B_{p}(1 / k), \quad p \in(0,1),
$$

where $N B_{p}(1 / k)$ is the $1 / k$-lag increment of the NBP. In [176, 180, the distribution of $N_{p}^{(k)}+1$, which is supported on the set of integers $\{1, k+1,2 k+1, \ldots\}$, is referred to as Harris law (see also [17, 141] in this connection). Since $N_{p}(k)$ is ID, it leads to a Lévy processes $\left\{N_{p}^{(k)}(t), t \geq 0\right\}$, which in turn allows us to define the family of such processes with drift,

$$
\mathcal{T}_{k}=\left\{T_{c}^{(k)}(t)=t+N_{p}^{(k)}(t), t \geq 0\right\}, \quad p \in(0,1), \quad c=1 / p \geq 1 .
$$

This family satisfies all the required properties, including 46 . Then the re-scaled standard gamma Lévy process $\left\{G^{(k)}(t), t \geq 0\right\}$, where $G^{(k)}(1)=k G(1 / k)$ (gamma distribution with shape parameter $1 / k$ and scale parameter $k$ ), is stochastically self-similar (with index $H=1$ ) with respect to the family $\mathcal{T}_{k}$ given by 499 . See [110] for more details and further generalizations. 
REMARK 6.5. As noted in [110, the Lévy process $\left\{G_{\alpha}(t), t \geq 0\right\}$, whose lag-1 increments have the Mittag-Leffler distribution given by the LT (4), is stochastically self-similar with index $H=1 / \alpha$ with respect to the family of negative binomial time changes (47).

Other stochastic self-similar processes can be constructed by subordinating stable processes to the gamma process or the Mittag-Leffler Lévy process $G_{\alpha}(t)$ discussed above. Here, one would follow the following general fact, noted in 94.

Proposition 6.6. If $\{X(t), t \geq 0\}$ is any self-similar process with index $H$ and $\{Z(t)$, $t \geq 0\}$ is an independent Lévy process that is stochastically self-similar with index $H^{\prime}$ with respect to a family $\mathcal{T}=\left\{T_{c}(t), t \geq 0\right\}, c \geq 1$, then the subordinated process $\{Y(t)=$ $X(Z(t)), t \geq 0\}$ is stochastically self-similar with index $H H^{\prime}$ with respect to the same family.

When $X(t)$ is a Brownian motion and $Z(t)$ is a gamma process, we obtain the Laplace motion discussed in [77. This model plays an increasingly important role in mathematical finance, where it is known as the variance-gamma process (see [21, 130, 131, 183]). A more general process with correlated increments, termed a fractional Laplace motion in [94, 138, arises by subordinating a fractional Brownian motion to the gamma process.

6.1.1. Fractional Laplace motion. Let $\left\{B_{H}(t), t \geq 0\right\}, H \in(0,1)$, be a fractional Brownian motion with the covariance function

$$
E\left[B_{H}(t) B_{H}(s)\right]=\frac{\sigma^{2}}{2}\left(|t|^{2 H}+|s|^{2 H}-|t-s|^{2 H}\right), t, s \geq 0,
$$

and let $\{G(t), t \geq 0\}$ be a standard gamma process. Then

$$
\{Y(t), t \geq 0\} \stackrel{d}{=}\left\{B_{H}(G(t)), t \geq 0\right\}
$$

is the fractional Laplace motion (FLM) with shape parameter 1, denoted by $\mathcal{F} \mathcal{L} \mathcal{M}_{H}(\sigma)$. (When $G$ in (51) is a gamma process with parameter $\nu>0$, which is a Lévy process whose lag- $t$ increments have gamma distributions with shape parameter $t / \nu$ and unit scale, we obtain a more general FLM with shape parameter $\nu>0$.) Since the gamma process is $H$-sss with $H=1$ with respect to the family (47), by Proposition 6.6, so is the FLM (51). The one-dimensional distributions of $\mathcal{F} \mathcal{L} \mathcal{M}_{H}(\sigma)$ are scale mixtures of Gaussian laws,

$$
Y(t) \stackrel{d}{=}\left(\sigma^{2}[G(t)]^{2 H}\right)^{1 / 2} Z .
$$

Here, $Z$ is a standard normal random variable while the "stochastic variance" $[G(t)]^{2 H}$, which is independent of $Z$, has the generalized gamma distribution given by the PDF

$$
f(x)=\frac{\gamma x^{\alpha \gamma-1}}{c^{\alpha \gamma} \Gamma(\alpha)} e^{-(x / c)^{\gamma}}, x>0,
$$

with scale $c=1$ and shape parameters $\alpha=t$ and $\gamma=0.5 / H$. It is worth noting that the distribution of $Y(t)$ is ID if and only if $1 / 2 \leq H \leq 1$, which reflects the fact that the powers of gamma variable $[G(t)]^{1 / \gamma}$ in $(52)$ are ID whenever $0<|\gamma| \leq 1$ but not ID for $\gamma>1$ (interestingly, the case $\gamma<-1$ is still open). This is closely related to the tail behavior of the FLM, derived in [94]. 
Proposition 6.7. The tail probability of $Y(t) \sim \mathcal{F} \mathcal{L M}_{H}(\sigma)$ admits the following asymptotic behavior:

$$
P(Y(t)>x) \sim c x^{2(t-1) /(1+2 H)} \exp \left\{-b x^{2 /(1+2 H)}\right\}, \quad x \rightarrow \infty,
$$

where $c=a H^{2 H /(1+2 H)}$ and

$$
a=\frac{1}{\sqrt{1+2 H} \Gamma(t)} H^{t /(1+2 H)-1 / 2}, \quad b=\frac{1+2 H}{2} H^{-2 H /(1+2 H)} .
$$

Other basic properties of the FLM, derived in [94], reflect those of the FBM. In particular, the covariance structures of the $\mathcal{F} \mathcal{L M}_{H}(\sigma)$ is asymptotically equivalent to that of the FBM. Similarly, the moment scaling of the $\mathcal{F} \mathcal{L} \mathcal{M}_{H}(\sigma)$,

$$
E|Y(t)|^{q}=c_{q} \frac{\Gamma(H q+t)}{\Gamma(t)}, c_{q}=\sqrt{\frac{2^{q}}{\pi}} \Gamma\left(\frac{1+q}{2}\right),
$$

which is linear in $t$ when $q=1 / H$, is asymptotically the same as that of the FBM, $E|Y(t)|^{q} \sim c_{q} t^{q H}$ as $t \rightarrow \infty$. This reflects the fact that the increments of the FLM become Gaussian with an increasing lag. In analogy with the FBM, the stationary process

$$
\left\{W_{j}, j=1,2, \ldots\right\} \stackrel{d}{=}\{Y(\eta j)-Y(\eta(j-1)), j=1,2, \ldots\}, \eta>0
$$

consisting of lag- $\eta$ increments of the FLM, is called a fractional Laplace noise (FLN). The covariance function of the FLN, derived in 94, has the same asymptotic behavior as that of the fractional Brownian noise,

$$
r(n)=E\left[W_{j} W_{j+n}\right] \sim \sigma^{2} H(2 H-1)(\eta)^{2 H} n^{2 H-2} \text { as } n \rightarrow \infty \text { and } H \neq 0.5,
$$

so that when $H>1 / 2$ the series $\sum_{n=1}^{\infty} r(n)$ diverges, and similarly to the fractional Brownian noise, the FLN exhibits a long-range dependence. More details on theory and applications of the FLM or FLN can be found in 94, 138, 147, 148.

6.2. Geometric renormalization group and self-similarity. As noted in [94, 110, stochastic self-similarity of FLM leads to random stability properties of FLN. Indeed, by taking $t=1$ in the relation 44, we find that $(1 / p)^{H} Y(1) \stackrel{d}{=} Y\left(N_{p}(1)\right)$, where $Y$ is an FLM $\mathcal{F} \mathcal{L} \mathcal{M}_{H}(\sigma)$ and $\left\{N_{p}(t)=N B_{p}(t)+t, t \geq 0\right\}$ is the negative binomial process with drift. Since $Y\left(N_{p}(1)\right)$ is the sum of the first $N_{p}(1)$ lag-1 increments of $Y$, this relation can be written as

$$
p^{H} \sum_{j=1}^{N_{p}} W_{j} \stackrel{d}{=} W_{1}
$$

where $N_{p}=N_{p}(1)$ is a geometric variable (2) and the $\left\{W_{j}\right\}$ are the increments (57) of $Y$ with $\eta=1$. Note that when $H=1 / 2$, then the $\left\{W_{j}\right\}$ are independent and Laplace distributed, so this is a generalization of geometric stability. This motivates the following definition (see [94]).

Definition 6.8. For each $p \in(0,1)$, let $\left\{N_{p}(t), t \geq 1\right\}$ be a NB Lévy process with drift. A composition group of transformations $\left\{T_{p}, \circ\right\}, p \in(0,1)$, of stationary sequences $W=\left\{W_{j}, j=1,2, \ldots\right\}$, defined by

$$
T_{p}: W \rightarrow T_{p} W=\left\{\left(T_{p} W\right)_{k}, k=1,2, \ldots\right\}
$$


where

$$
\left(T_{p} W\right)_{k}=p^{H}\left(W_{N_{p}(k-1)+1}+\cdots+W_{N_{p}(k)}\right), k=1,2, \ldots
$$

and $N_{p}(t)$ is independent of $W$, is called a geometric renormalization group with parameter $H \in(0,1)$.

Thus, $T_{p}$ transforms a stationary sequence $W$ into another one, obtained by summing the components of $W$ over successive blocks of geometric size $N_{p}^{(k)}=N_{p}(k)-N_{p}(k-1)$ and normalizing by $p^{H}=(\text { expected block size })^{H}$. Note that this group is commutative, since we have $N_{p}\left(N_{q}(k)\right)=N_{q}\left(N_{p}(k)\right), p, q \in(0,1), k=1,2, \ldots$, where $N_{p}$ and $N_{q}$ are two independent negative binomial processes with drift. The fact that FLN is a fixed point of the transformations in in group, leads to the following definition (see [94])

Definition 6.9. Let $\left\{T_{p}, \circ\right\}, p \in(0,1)$, be a geometric renormalization group with parameter $H \in(0,1)$. If $W=\left\{W_{j}, j=1,2, \ldots\right\}$ is a stationary sequence and $T_{p} W \stackrel{d}{=} W$, $p \in(0,1)$, then we say that $W$ is geometrically self-similar with index $H$.

We see that a lag-1 FLN is a geometric self-similar stationary sequence. We conclude by noting that the notion of geometric self-similarity in relation to stationary sequences is quite similar to invariance properties of renewal processes with respect to geometric thinning, discussed in Section 2, since both properties are based on stability with respect to geometric summation.

Acknowledgements. The author thanks the referee for helpful comments.

\section{References}

[1] J. Andĕl, Marginal distributions of autoregressive processes, in: Transactions of the 9th Prague Conference on Information Theory, Statistical Decision Functions, Random Processes, Dordrecht, 1983, 127-135.

[2] E.-E. A. A. Aly and N. Bouzar, On geometric infinite divisibility and stability, Ann. Inst. Statist. Math. 52 (4) (2000), 790-799.

[3] E.-E. A. A. Aly and N. Bouzar, Stationary solutions for integer-valued autoregressive processes, Int. J. Mathematics Math. Sciences 2005 (1) (2005), 1-18.

[4] D. N. Anderson, A multivariate Linnik distribution, Statist. Probab. Lett. 14 (1992), 333-336.

[5] D. N. Anderson and B. C. Arnold, Linnik distributions and processes, J. Appl. Probab. 30 (1993), 330-340.

[6] R. B. Arellano-Valle, H. W. Gómez, and F. A. Quintana, Statistical inference for a general class of asymmetric distributions, J. Statist. Plan. Inference 128 (2005), 427-443.

[7] B. C. Arnold, Some characterizations of the exponential distribution by geometric compounding, SIAM J. Appl. Math. 24 (2) (1973), 242-244.

[8] A. Ayebo and T. J. Kozubowski, An asymmetric generalization of Gaussian and Laplace laws, J. Probab. Statist. Sci. 1 (2) (2003), 187-210.

[9] L. Baringhaus and R. Grübel, On a class of characterization problems for random complex combinations, Ann. Inst. Statist. Math. 49 (3) (1997), 555-567. 
[10] B. P. Belinskiy and T. J. Kozubowski, Exponential mixture representation of geometric stable densities, J. Math. Anal. Appl. 246 (2000), 465-479.

[11] F. Biondi, T. J. Kozubowski and A. K. Panorska, A new model for quantifying climate episodes, Intern. J. Climatology 25 (2005), 1253-1264.

[12] F. Biondi, T. J. Kozubowski, A. K. Panorska and L. Saito, A new stochastic model of episode peak and duration for eco-hydro-climatic applications, Ecological Modelling 211 (2008), 383-395.

[13] M. Borowiecka, Geometrically semistable distributions and a functional equation, J. Math. Sci. 111 (3) (2002), 3524-3527.

[14] N. Bouzar, On geometric stability and Poisson mixtures, Illinois J. Math. 43 (3) (1999), $520-527$.

[15] N. Bouzar, Discrete semi-stable distribution, Ann. Inst. Statist. Math. 56 (3) (2004), 497-510.

[16] J. Bunge, Some stability classes for random numbers of random vectors, Comm. Statist. Stochastic Models 9 (2) (1993), 247-253.

[17] J. Bunge, Composition semigroups and random stability, Ann. Probab. 24 (3) (1996), 1476-1489.

[18] S. Cambanis and A. Taraporevala, Infinitely divisible distributions with stable marginals (1994), preprint.

[19] D. Chenyao, S. Mittnik and S. T. Rachev, Distribution of exchange rates: A geometric summation-stable model, in: Proceedings of the Seminar on Data Analysis, Sept. 12-17, Sozopol, Bulgaria, 1996.

[20] G. Christoph and K. Schreiber, The generalized discrete Linnik distributions, in: Advances in Stochastic Models for Reliability, Quality, and Safety, W. Kahle et al. (eds.), Boston, Birkhäuser, 1998, 3-18.

[21] R. Cont and P. Tankov, Financial Modelling with Jump Processes, Chapman \& Hall/CRC, Boca Raton, 2004.

[22] L. S. Dewald and P. A. W. Lewis, A new Laplace second-order autoregressive time series model-NLAR(2), IEEE Trans. Inform. Theory 31 (5) (1985), 645-651.

[23] L. Devroye, A note on Linnik distribution, Statist. Probab. Lett. 9 (1990), 305-306.

[24] L. Devroye, A triptych of discrete distributions related to the stable law, Statist. Probab. Lett. 18 (1993), 349-351.

[25] L. Devroye, Random variate generation in one line code, in: Proc. Winter Simulation Conf., IEEE Press, New York, 1996, 265-272.

[26] G. Divanji, On semi- $\alpha$-Laplace distributions, J. Indian Statist. Assoc. 26 (1988), 31-38.

[27] R. L. Dobrushin, A lemma on the limit of a compound random function, Uspekhi Mat. Nauk 10 (1955), 157.

[28] P. Embrechts and M. Maejima, Selfsimilar Processes, Princeton University Press, Princeton and Oxford, 2002.

[29] M. B. Erdogan, Analytic and asymptotic properties of non-symmetric Linnik's probability densities, PhD Thesis, Bilkent University, Ankara, 1995; appeared in J. Fourier Anal. Appl. 5 (6) (1999), 523-544.

[30] M. B. Erdogan and I. V. Ostrovskii, Non-symmetric Linnik distributions, C. R. Acad. Sci. Paris 325, Série I (1997), 511-516.

[31] M. B. Erdogan and I. V. Ostrovskii, On mixture representation of the Linnik density, J. Austral. Math. Soc. Ser. A 64 (1998), 317-326. 
[32] M. B. Erdogan and I. V. Ostrovskii, Analytic and asymptotic properties of generalized Linnik probability densities, J. Math. Anal. Appl. 217 (1998), 555-579.

[33] C. Fernandez, J. Osiewalski, and M. F. J. Steel, Modelling and inference with v-distributions, J. Amer. Statist. Assoc. 90 (1995), 1331-1340.

[34] C. Fernandez and M. F. J. Steel, On Bayesian modeling of fat tails and skewness, J. Amer. Statist. Assoc. 93 (1998), 359-371.

[35] D. G. Gaver and P. A. W. Lewis, First order autoregressive gamma sequences and point processes, Adv. Appl. Probab. 12 (1980), 727-745.

[36] W. Gawronski, On the unimodality of geometric stable laws, Statist. Decisions 19 (4) (2001), 405-417.

[37] B. V. Gnedenko, Limit theorems for sums of random number of positive independent random variables, Proc. 6th Berkeley Symp. on Math. Statist. Probab. 2 (1970), 537-549.

[38] B. V. Gnedenko, On limit theorems for a random number of random variables, in: Probability Theory and Mathematical Statistics. Fourth USSR-Japan Symposium Proceedings, K. Itô and J. V. Prokhorov (eds.), Lecture Notes in Math. 1021, Springer-Verlag, 1982, $167-176$.

[39] B. V. Gnedenko, On limit theorems for a random number of random variables, Lecture Notes in Math. 1021 (1983), 167.

[40] B. V. Gnedenko and H. Fakhim, On a transfer theorem, Doklady Acad. Nauk USSR 187 (1969), 15-17 (in Russian).

[41] B. V. Gnedenko and S. Janjič, A characteristic property of one class of limit distributions, Math. Nachr. 113 (1983), 145-149.

[42] B. V. Gnedenko and V. Yu. Korolev, Random Summation. Limit Theorems and Applications, CRC Press, Boca Raton, 1996.

[43] B. V. Gnedenko and I. N. Kovalenko, Introduction to Queueing Theory, Israel Program for Scientific Translation, Jerusalem, 1968.

[44] H. W. Gómez, F. J. Torres, and H. Bolfarine, Large-sample inference for the epsilonskew-t distribution, Comm. Statist. Theory Methods 36 (2007), 73-81.

[45] R. Gorenflo and F. Mainardi, Continuous time random walk, Mittag-Leffler waiting time and fractional diffusion: mathematical aspects, in: Anomalous Transport: Foundations and Applications, R. Klages, G. Radons, and I. M. Sokolov (eds.), Wiley-VCH, Weinheim, 2008, 93-129.

[46] V. K. Gupta and E. C. Waymire, Multiscaling properties of spatial rainfall in river flow distributions, J. Geophys. Res. 95 (1990), 1999-2009.

[47] M. J. Hartley and N. S. Revankar, On the estimation of the Pareto law from underreported data, J. Econometrics 2 (1974), 327-341.

[48] W. Hazod, On the limit behavior of products of a random number of group valued random variables, Theory Probab. Appl. 32 (1995), 249-263.

[49] W. Hazod, On geometric convolutions of distributions of group-valued random variables, in: Probability Measures on Groups and Related Structures, Proceedings Oberwolfach 1994, World Scientific, 1995, 167-181.

[50] W. Hazod and Yu. S. Khokhlov, On Szasz's compactness theorem and applications to geometric stability on groups, Prob. Math. Stat. 16 (1996), 143-156.

[51] W. Hazod and E. Siebert, Stable Probability Measures on Euclidean Spaces and on Locally Compact Groups, Kluwer Academic Publishers, 2001.

[52] D. V. Hinkley and N. S. Revankar, Estimation of the Pareto law from underreported data, J. Econometrics 5 (1977), 1-11. 
[53] B. V. Huff, The strict subordination of differential processes, Sankhya Ser. A 31 (1969), 403-412.

[54] S. Janjić, On random variables with the same distribution type as their random sum, Publ. Inst. Math. (Beograd) N.S. 35 (49) (1984), 161-166.

[55] S. Jankovič, Preservation of type under mixing, Teor. Veroyatnost. i Primenen. 37 (1992), 594-599.

[56] S. Jankovič, Enlargement of the class of geometrically infinitely divisible random variables, Publ. Inst. Math. (Beograd) (N.S.) 54 (68) (1993), 126-134.

[57] K. Jayakumar, The stationary solution of a first order integer-valued autoregressive process, Statistica 55 (1995), 221-228.

[58] K. Jayakumar, K. Kalyanaraman, and R. N. Pillai, $\alpha$-Laplace processes, Math. Comput. Modelling 22 (1) (1995), 109-116.

[59] K. Jayakumar and A. P. Kuttykrishnan, A time-series model using asymmetric Laplace distribution, Statist. Probab. Lett. 77 (16) (2007), 1636-1640.

[60] K. Jayakumar and R. N. Pillai, On class L. distributions, J. Indian Statist. Assoc. (30) (1992), 103-108.

[61] K. Jayakumar and R. N. Pillai, The first-order autoregressive Mittag-Leffler process, J. Appl. Probab. 30 (1993), 462-466.

[62] K. Jayakumar and R. N. Pillai, Characterization of Mittag-Leffler distribution, J. Appl. Statist. Sci. 4 (1) (1996), 77-82.

[63] K. Jayakumar and R. P. Suresh, Mittag-Leffler distributions, J. Indian Soc. Probab. Statist. 7 (2003), 51-71.

[64] Z. Jurek and J. D. Mason, Operator-Limit Distributions in Probability Theory, Wiley, New York, 1993.

[65] V. Jurić and T. J. Kozubowski, Skew Weibull distributions on the real line I: Basic properties, J. Probab. Statist. Sci. 2 (2) (2004), 187-198.

[66] V. Jurić and T. J. Kozubowski, Skew Weibull distributions on the real line II: Estimation and applications, J. Probab. Statist. Sci. 3 (1) (2005), 43-58.

[67] A. V. Kakosyan, L. B. Klebanov, and J. A. Melamed, Characterizations of Distributions by the Method of Intensively Monotone Operators, Lecture Notes in Mathematics 1088, Springer-Verlag, New York, 1984.

[68] V. Kalashnikov Geometric Sums: Bounds for Rare Events with Applications, Kluwer Acad. Publ., Dordrecht, 1997.

[69] L. B. Klebanov, T. J. Kozubowski, and S. T. Rachev, Ill-Posed Problems in Probability and Stability of Random Sums, Nova Science Publishers, New York, 2006.

[70] L. B. Klebanov, G. M. Maniya, and I. A. Melamed, A problem of Zolotarev and analogs of infinitely divisible and stable distributions in a scheme for summing a random number of random variables, Theory Probab. Appl. 29 (1984), 791-794.

[71] L. B. Klebanov, G. M. Maniya, and J. M. Melamed, Analogs of infinitely divisible and stable laws for sums of random number of random variables, Fourth Intern. Vilnius Conference on the Theory of Probability and Mathematical Statistics 2 (1985), 40-41 (in Russian).

[72] L. B. Klebanov, G. M. Maniya, and J. M. Melamed, $\nu_{p}$-strictly stable laws and the estimation of their parameters, Lecture Notes in Math. 1233, Springer, Berlin, 1987, 21-31.

[73] L. B. Klebanov, J. A. Melamed, S. Mittnik, and S. T. Rachev, Integral and asymptotic representations of geo-stable densities, Appl. Math. Lett. 9 (6) (1996), 37-40. 
[74] L. B. Klebanov, J. A. Melamed, and S. T. Rachev, On the products of a random number of random variables in connection with a problem from mathematical economics, in: Lect. Notes Math. 1412, Springer-Verlag, Berlin, 1989, 103-109.

[75] L. B. Klebanov and S. T. Rachev, Sums of random number of random variables and their approximations with $\nu$-accompanying infinitely divisible laws, Serdica Math. J. 22 (1996), 471-496.

[76] I. Komunjer, Asymmetric power distribution: theory and applications to risk measurement, J. Appl. Econ. 22 (2007), 891-921.

[77] S. Kotz, T. J. Kozubowski, and K. Podgórski, The Laplace Distribution and Generalizations: A Revisit with Applications to Communications, Economics, Engineering, and Finance, Birkhäuser, Boston, 2001.

[78] S. Kotz and I. V. Ostrovskii, A mixture representation of the Linnik distribution, Statist. Probab. Lett. 26 (1996), 61-64.

[79] S. Kotz, I. V. Ostrovskii, and A. Hayfavi, Analytic and asymptotic properties of Linnik's probability densities, I and II, J. Math. Anal. Appl. 193 (1995), 353-371 and 497-521.

[80] I. N. Kovalenko, On the class of limit distributions for thinning streams of homogeneous events, Litovsk. Mat. Sb. 5 (1965), 569-573 (Russian. Lithuanian, English summaries).

[81] T. J. Kozubowski, The theory of geometric stable distributions and its use in modeling financial data, Ph.D. Dissertation, University of California, Santa Barbara, 1992.

[82] T. J. Kozubowski, Estimation of the parameters of geometric stable laws, Technical Report No. 253, Department of Statistics and Applied Probability, University of California, Santa Barbara, 1993; appeared in Math. Comput. Modelling 29 (1999), 241-253.

[83] T. J. Kozubowski, Representation and properties of geometric stable laws, in: Approximation, Probability, and Related Fields, G. Anastassiou and S. T. Rachev (eds.), Plenum, New York, 1994, 321-337.

[84] T. J. Kozubowski, The inner characterization of geometric stable laws, Statist. Decisions 12 (1994), 307-321.

[85] T. J. Kozubowski, Characterization of multivariate geometric stable distributions, Statist. Decisions 15 (1997), 397-416.

[86] T. J. Kozubowski, Mixture representation of Linnik distribution revisited, Statist. Probab. Lett. 38 (1998), 157-160.

[87] T. J. Kozubowski, Exponential mixture representation of geometric stable distributions, Ann. Inst. Statist. Math. 52 (2) (2000), 231-238.

[88] T. J. Kozubowski, Computer simulation of geometric stable random variables, J. Comp. Appl. Math. 116 (2000), 221-229.

[89] T. J. Kozubowski, Fractional moment estimation of Linnik and Mittag-Leffler parameters, Math. Comput. Modelling 34 (2001), 1023-1035.

[90] T. J. Kozubowski, Infinite divisibility of Mittag-Leffler laws, Far East J. Theoret. Statis. 1 (6) (2002), 1-3.

[91] T. J. Kozubowski and M. M. Meerschaert, A bivariate infinitely divisible distribution with exponential and Mittag-Leffler marginals, Statist. Probab. Lett. 79 (14) (2009), 1596-1601.

[92] T. J. Kozubowski, M. M. Meerschaert, A. K. Panorska, and H.-P. Scheffler, Operator geometric stable laws, J. Multivariate Anal. 92 (2005), 298-323.

[93] T. J. Kozubowski, M. M. Meerschaert, and H.-P. Scheffler, The operator $\nu$-stable laws, Publ. Math. Debrecen 63 (4) (2003), 569-585.

[94] T. J. Kozubowski, M. M. Meerschaert, and K. Podgórski, Fractional Laplace Motion, Adv. Appl. Probab. 38 (2006), 451-464. 
[95] T. J. Kozubowski and A. K. Panorska, On moments and tail behavior of $\nu$-stable random variables, Statist. Probab. Lett. 29 (1996), 307-315.

[96] T. J. Kozubowski and A. K. Panorska, Weak limits for multivariate random sums, J. Multivariate Anal. 67 (1998), 398-413.

[97] T. J. Kozubowski and A. K. Panorska, Multivariate geometric stable distributions in financial applications, Math. Comput. Modelling 29 (1999), 83-92.

[98] T. J. Kozubowski and A. K. Panorska, Simulation of geometric stable and other limiting multivariate distributions arising in random summation scheme, Math. Comput. Modelling 29 (1999), 255-262.

[99] T. J. Kozubowski and A. K. Panorska, A mixed bivariate distribution with exponential and geometric marginals, J. Statist. Plann. Inference 134 (2005), 501-520.

[100] T. J. Kozubowski and A. K. Panorska, A mixed bivariate distribution connected with geometric maxima of exponential variables, Comm. Statist. Theory Methods 37 (2008), 2903-2923.

[101] T. J. Kozubowski, A. K. Panorska, and F. Biondi, Mixed multivariate models for random sums and maxima, in: Advances in Multivariate Statistical Methods, A. SenGupta (ed.), Statistical Science and Interdisciplinary Research Vol. 4, World Scientific, Singapore, 2009, 145-171.

[102] T. J. Kozubowski, A. K. Panorska and K. Podgórski, A bivariate Levy process with negative binomial and gamma marginals, J. Multivariate Anal. 99 (2008), 1418-1437.

[103] T. J. Kozubowski and K. Podgórski, A class of asymmetric distributions, Actuarial Research Clearing House 1 (1999), 113-134.

[104] T. J. Kozubowski and K. Podgórski, Geometric stable laws through series representations, Serdica Math. J. 25 (1999), 241-256.

[105] T. J. Kozubowski and K. Podgórski, Asymmetric Laplace distributions, Math. Sci. 25 (2000), 37-46.

[106] T. J. Kozubowski and K. Podgórski, A multivariate and asymmetric generalization of Laplace distribution, Comput. Statist. 15 (2000), 531-540.

[107] T. J. Kozubowski and K. Podgórski, Asymmetric Laplace laws and modeling financial data, Math. Comput. Modelling 34 (2001), 1003-1021.

[108] T. J. Kozubowski and K. Podgórski, Log-Laplace distributions, Internat. Math. J. 3 (2003), 467-495.

[109] T. J. Kozubowski and K. Podgórski, A log-Laplace growth rate model, Math. Sci. 28 (2003), 49-60.

[110] T. J. Kozubowski and K. Podgórski, Invariance properties of the negative binomial Lévy process and stochastic self-similarity, Int. Math. Forum 2 (30) (2007), 1457-1468.

[111] T. J. Kozubowski and K. Podgórski, Skewed Laplace distributions I: their origins and inter-relations, Math. Sci. 33 (2008), 22-34.

[112] T. J. Kozubowski and K. Podgórski, Skewed Laplace distributions II: divisibility properties and extensions to stochastic processes, Math. Sci. 33 (2008), 35-48.

[113] T. J. Kozubowski and K. Podgórski, Distributional properties of the negative binomial Lévy process, Probab. Math. Statist. 29 (2009), 43-71.

[114] T. J. Kozubowski and K. Podgórski, Rational characteristic functions and geometric infinite divisibility, J. Math. Anal. Appl. 365 (2010) 625-637.

[115] T. J. Kozubowski, K. Podgórski, and G. Samorodnitsky, Tails of Lévy measure of geometric stable random variables, Extremes 1 (3) (1998), 367-378.

[116] T. J. Kozubowski and S. T. Rachev, The theory of geometric stable distributions and its use in modeling financial data, European J. Oper. Res. 74 (1994), 310-324. 
[117] T. J. Kozubowski and S. T. Rachev, Univariate geometric stable laws, J. Comput. Anal. Appl. 1 (2) (1999), 177-217.

[118] T. J. Kozubowski and S. T. Rachev, Multivariate geometric stable laws, J. Comput. Anal. Appl. 1 (4) (1999), 349-385.

[119] V. M. Kruglov, On the extension of the class of stable distributions, Theory Probab. Appl. 17 (1972), 685-694.

[120] V. M. Kruglov and V. Yu. Korolev, Limit Theorems For Random Sums, Izdat. Mosk. Univ., Moscow, 1990 (in Russian).

[121] A. J. Lawrence, Some autoregressive models for point processes, in: Point Processes and Queuing Problems (Keszthely, 1978), Colloq. Math. Soc. János Bolyai, 24, NorthHolland, Amsterdam-New York, 1981, 257-275.

[122] A. J. Lawrence and P. A. W. Lewis, An exponential autoregressive-moving average process, EARMA(p,q), J. Roy. Statist. Soc. B 42 (2) (1980), 150-161.

[123] A. J. Lawrence and P. A. W. Lewis, A new autoregressive time series model in exponential variates (NEAR(1)), Adv. Appl. Probab. 13 (1981), 826-845.

[124] M.-L. T. Lee, S. T. Rachev, and G. Samorodnitsky, Dependence of stable random variables, in: Stochastic Inequalities, M. Shaked and Y. L. Tong (eds.), IMS Lecture Notes - Monograph Series 22, IMS, Hayward, 219-234.

[125] G. D. Lin, Characterizations of the Laplace and related distributions via geometric compound, Sankhya Ser. A 56 (1994), 1-9.

[126] G. D. Lin, On the Mittag-Leffler distributions, J. Statist. Plann. Inference 74 (1998), 1-9.

[127] G. D. Lin, A note on the Linnik distributions, J. Math. Anal. Appl. 217 (1998), 701-706.

[128] G. D. Lin, A note on the characterization of positive Linnik laws, Aust. N. Z. J. Statist. 43 (1) (2001), 17-20.

[129] Yu. V. Linnik, Linear forms and statistical criteria, I, II, Selected Translations in Math. Statist. Probab. 3 (1963), 1-90. (The original paper appeared in Ukrainskii Mat. Zhurnal 5 (1953), 207-290.)

[130] D. B. Madan, P. P. Carr, and E. C. Chang, The variance gamma process and option pricing, European Finance Rev. 2 (1998), 79-105.

[131] D. B. Madan and E. Seneta, The variance gamma (V. G.) model for share markets returns, J. Business 63 (1991), 511-524.

[132] M. Maejima and J. Rosiński, The class of type $G$ distributions on $R^{d}$ and related subclasses of infinitely divisible distributions, Demonstratio Math. 34 (2) (2001), 251-266.

[133] M. Maejima and J. Rosiński, The class of type $G$ distributions on $R^{d}$, J. Theoret. Probab. 15 (2) (2002), 323-341.

[134] M. T. Malinowski, Geometric strictly semistable laws as the limit laws, Discuss. Math. Probab. Statist. 27 (2007), 79-97.

[135] M. T. Malinowski, On some limit distributions for geometric random sums, Discuss. Math. Probab. Statist. 28 (2008), 247-266.

[136] M. B. Marcus, $\xi$-radial Processes and Random Fourier Series, Mem. Ser. 68, Amer. Math. Soc., Providence, 1987.

[137] M. M. Meerschaert, Regular variation in $R^{k}$ and vector-normed domains of attraction, Statist. Probab. Lett. 11 (1991), 287-289.

[138] M. M. Meerschaert, T. J. Kozubowski, F. Molz and S. Lu, Fractional Laplace model for hydraulic conductivity, Geophysical Res. Lett. 31 (2004), L08501.

[139] M. M. Meerschaert and H. P. Scheffler, Limit Distributions for Sums of Independent Random Vectors, Wiley, New York, 2001. 
[140] M. M. Meerschaert and H. P. Scheffler, Portfolio modeling with heavy tailed random vectors, in: Handbook of Heavy Tailed Distributions in Finance, S. T. Rachev (ed.), Elsevier Science, Amsterdam, 2003, 595-640.

[141] I. A. Melamed, Inequalities for the moments of $\nu$-infinitely divisible laws and the characterization of probability distributions, J. Soviet Math. 59 (1992), 960-970.

[142] S. Mittnik and S. T. Rachev, Alternative multivariate stable distributions and their applications to financial modelling, in: Stable Processes and Related Topics, S. Cambanis et al. (eds.), Birkhäuser, Boston, 1991, 107-119.

[143] S. Mittnik and S. T. Rachev, Modeling asset returns with alternative stable distributions, Econometric Rev. 12 (3) (1993), 261-330.

[144] S. Mittnik and S. T. Rachev, Reply to comments on "Modeling asset returns with alternative stable distributions" and some extensions, Econometric Rev. 12 (3) (1993), 347-389.

[145] S. Mittnik, S. T. Rachev and L. Rüschendorf, Test of association between multivariate stable vectors, Math. Comput. Modelling 29 (1999), 181-195.

[146] N. R. Mohan, R. Vasudeva and H. V. Hebbar, On geometrically infinitely divisible laws and geometric domains of attraction, Sankhya Ser. A 55 (1993), 171-179.

[147] F. J. Molz, T. J. Kozubowski, K. Podgórski, and J. W. Castle, A generalization of the fractal/facies model, Hydrogeology Journal 15 (2007), 809-816.

[148] F. J. Molz, M. M. Meerschaert, T. J. Kozubowski and P. D. Hyden, Do heterogeneous sediment properties and turbulent velocity fluctuations have something in common? Some history and a new stochastic process, in: Dynamics of Fluids and Transport in Fractured Rock, B. Faybishenko and P. A. Witherspoon (eds.), AGU Monograph 162, American Geophysical Union, Washington, DC, 2005, 13-22.

[149] G. S. Mudholkar and A. D. Hutson, The epsilon-skew-normal distribution for analyzing near-normal data, J. Statist. Plann. Inference 83 (2000), 291-309.

[150] I. V. Ostrovskii, Analytic and asymptotic properties of multivariate Linnik's distribution, Mathematical Physics, Analysis, Geometry 2 (3/4) (1995), 436-455.

[151] A. G. Pakes, Characterization of discrete laws via mixed sums and Markov branching processes, Stoch. Process. Appl. 55 (1995), 285-300.

[152] A. K. Pakes, Mixture representations for symmetric generalized Linnik laws, Statist. Probab. Lett. 37 (1998), 213-221.

[153] R. N. Pillai, Semi-stable laws as limit distributions, Ann. Math. Statist. 42 (1971), 780783.

[154] R. N. Pillai, Semi- $\alpha$-Laplace distributions, Comm. Statist. Theory Methods 14 (4) (1985), 991-1000.

[155] R. N. Pillai, Harmonic mixtures and geometric infinite divisibility, J. Indian Statist. Assoc. 28 (1990), 87-98.

[156] R. N. Pillai, On Mittag-Leffler functions and related distributions, Ann. Inst. Statist. Math. 42 (1) (1990), 157-161.

[157] R. N. Pillai and K. Jayakumar, Discrete Mittag-Leffler distributions, Statist. Probab. Lett. 23 (1995), 271-274.

[158] R. N. Pillai and E. Sandhya, Distributions with complete monotone derivative and geometric infinite divisibility, Adv. Appl. Probab. 22 (1990), 751-754.

[159] S. T. Rachev and S. Mittnik, Stable Paretian Models in Finance, Wiley, Chichester, 2000.

[160] S. T. Rachev and G. Samorodnitsky, Geometric stable distributions in Banach spaces, J. Theoret. Probab. 7 (2) (1994), 351-373. 
[161] S. T. Rachev and A. SenGupta, Geometric stable distributions and Laplace-Weibull mixtures, Statist. Decisions 10 (1992), 251-271.

[162] S. T. Rachev and A. SenGupta, Laplace-Weibull mixtures for modeling price changes, Management Science 39 (8) (1993), 1029-1038.

[163] B. Ramachandran, On geometric stable laws, a related property of stable processes, and stable densities, Ann. Inst. Statist. Math. 49 (2) (1997), 299-313.

[164] W. J. Reed, The Pareto, Zipf and other power laws, Econ. Lett. 4 (2001), 15-19.

[165] A. Rényi, A characterization of Poisson processes, in: Selected Papers of Alfréd Rényi, P. Turán (ed.) 1, Akadémiai Kiadó, Budapest, 1976, 622-628.

[166] S. Resnick and P. Greenwood, A bivariate stable characterization and domains of attraction, J. Multivariate Anal. 9 (1979), 206-221.

[167] H. Robbins, Mixtures of distributions, Ann. Math. Statist. 19 (1948), 360-369.

[168] H. Robbins, On the asymptotic distribution of the sum of a random number of random variables, Proc. Nat. Acad. Sci. 34 (1948), 162-163.

[169] J. Rosiński, Weak compactness of laws of random sums of identically distributed random vectors in Banach spaces, Colloq. Math. 35 (2) (1976), 313-325.

[170] J. Rosiński, On a class of infinitely divisible processes represented as mixtures of Gaussian processes, in: Stable Processes and Related Topics, S. Cambanis, G. Samorodnitsky, and M. S. Taqqu (eds.), Birkhäuser, Basel, 1991, 27-41.

[171] Z. Rychlik and D. Szynal, On the limit behavior of a random number of independent random variables, Coll. Math. 28 (1973), 147-159.

[172] G. Samorodnitsky and M. Taqqu, Stable Non-Gaussian Random Processes, Chapman \& Hall, New York, 2001.

[173] E. Sandhya, Geometric Infinite Divisibility and Applications, Ph.D. Thesis, University of Kerala, 1991.

[174] E. Sandhya and R. N. Pillai, On geometric infinite divisibility, J. Kerala Statist. Assoc. 10 (1999), 1-7.

[175] E. Sandhya and R. N. Pillai, Renewal theory and geometric infinite divisibility, ProbStat Models 2 (2003), 1-8.

[176] S. Satheesh, Stability of random sums and extremes, Ph.D. Thesis, Cochin University of Science and Technology, 2001.

[177] S. Satheesh, Aspects of randomization in infinitely divisible and max-infinitely divisible laws, ProbStat Models 1 (June 2002), 7-16.

[178] S. Satheesh, Another look at random infinite divisibility, Statist. Methods 6 (2) (2004), 123-144.

[179] S. Satheesh and N. U. Nair, Some classes of distributions on the non-negative lattice, J. Indian Statist. Assoc. 40 (1) (2002), 41-58.

[180] S. Satheesh, N. U. Nair, and E. Sandhya, Stability of random sums, Stochastic Modelling and Applications 5 (1) (2002), 17-26.

[181] S. Satheesh and E. Sandhya, Infinite divisibility and max-infinite divisibility with random sample size, Statist. Methods 5 (2) (2003), 126-139.

[182] K. I. Sato, Lévy Processes and Infinitely Divisible Distributions, Cambridge University Press, 1999.

[183] W. Schoutens, Lévy Processes in Finance: Pricing Financial Derivatives, John Wiley \& Sons, Chichester, 2003.

[184] V. Seetha Lekshmi and K. K. Jose, An autoregressive process with geometric $\alpha$-Laplace marginals, Statist. Papers 45 (2004), 337-350. 
[185] V. Seetha Lekshmi and K. K. Jose, Geometric Mittag-Leffler distributions and processes, J. Appl. Statist. 13 (2004), 335-342.

[186] V. Seetha Lekshmi and K. K. Jose, Autoregressive processes with Pakes and geometric Pakes generalized Linnik marginals, Statist. Probab. Lett. 76 (2006), 318-326.

[187] V. Seetha Lekshmi, J. Jacob and K. K. Jose, Generalized Laplacian and geometric $\alpha-$ Laplace distributions with applications in time series modelling, Statist. Meth. 5 (2003), 140-155.

[188] F. W. Steutel and K. van Harn, Discrete analogs of self-decomposability and stability, Ann. Probab. 7 (5) (1979), 893-899.

[189] T. Szántai, On limiting distributions for the sums of random number of random variables concerning the rarefaction of recurrent process, Studia Sci. Math. Hungar. 6 (1971), 443452.

[190] D. Szasz, On classes of limit distributions for sums of a random number of identically distributed independent random variables, Theory Probab. Appl. 27 (1972), 401-415.

[191] D. Veneziano, Basic properties and characterization of stochastically self-similar processes in $R^{d}$, Fractals 7 (1999), 59-78.

[192] K. Weron and M. Kotulski, On the Cole-Cole relaxation function and related MittagLeffler distributions, Physica A 232 (1996), 180-188. 
